\title{
Philosophiques
}

\section{Il y a toujours un mort celé dans le miroir : structure spéculaire et métaphores tragiques dans D'un miroir et de quelques éclats de Pierre Gravel \\ Pierre Gravel, D’un miroir et de quelques éclats, Montréal, L'Hexagone, coll. Positions Philosophiques, 1985, 179 p.}

\section{Michael La Chance}

Volume 13, numéro 2, automne 1986

URI : https://id.erudit.org/iderudit/203326ar

DOI : https://doi.org/10.7202/203326ar

Aller au sommaire du numéro

Éditeur(s)

Société de philosophie du Québec

ISSN

0316-2923 (imprimé)

1492-1391 (numérique)

Découvrir la revue

Citer cet article

La Chance, M. (1986). Il y a toujours un mort celé dans le miroir : structure spéculaire et métaphores tragiques dans D'un miroir et de quelques éclats de Pierre Gravel / Pierre Gravel, D'un miroir et de quelques éclats, Montréal, L'Hexagone, coll. Positions Philosophiques, 1985, 179 p. Philosophiques, 13(2), 369-382. https://doi.org/10.7202/203326ar d'utilisation que vous pouvez consulter en ligne. 


\title{
ÉTUDES CRITIQUES
}

\section{IL Y A TOUJOURS UN MORT CELÉ DANS \\ LE MIROIR : STRUCTURE SPÉCULAIRE ET MÉTAPHORES \\ TRAGIQUES DANS D'UN MIROIR ET DE QUELQUES ÉCLATS DE PIERRE GRAVEL}

PIERRE GRAVEL, D'un miroir et de quelques éclats, Montréal, L'Hexagone, coll. Positions Philosophiques, 1985, 179 p.

par Michael La Chance.

\begin{abstract}
L'impropre
... nous avons plaisir à regarder les images les plus soignées des choses dont la vue nous est pénible dans la réalité, par exemple les formes d'animaux parfaitement ignobles ou de cadavres; la raison en est qu'apprendre est un plaisir non seulement pour les philosophes, mais également pour les autres hommes (mais ce qu'il y a de commun entre eux sur ce point se limite à peu de choses);
\end{abstract}

Aristote, Poétique 1

\section{HORREUR ET REPRÉSENTATION}

Nous avons l'attrait de l'horreur, parce que le regard s'y éteint. Dans la représentation, l'horreur est soutenue et nous gardons les yeux ouverts. Peut-être aussi croyons-nous voir au-delà. Le complexe et l'horrifiant sont résorbés dans le muthos mais y sont d'une certaine façon répétés. C'est la question du pouvoir représentatif de la philosophie tragique qui se posait avant Socrate et que nous redécouvrons aujourd'hui avec la fin d'une modernité: celle d'une histoire inhumaine devant laquelle les schémas narratifs ne tiennent plus. Comment dès lors l'homme moderne peut-il se construire une identité?

Le propos de Pierre Gravel s'inscrit dans ce désir de renouer avec une philosophie tragique. Avant d'y souscrire il faut se demander si ce n'est pas un certain système de représentation, par les zones d'ombres et de lumières qu'il dessine, qui produit l'horreur dont il doit précisément être le contrejour : les plaies, difformités et cadavres évoqués à tout instant au détour de

1. Trad. R. Dupont-Roc et J. Lallot, Seuil, 1980, 48b10-16. 
la réflexion signalent bien cet au-delà de la pensée par rapport auquel elle s'institue et se met en scène. Il s'agit de l'horreur extrême du sans-nom, de l'identité absente, du vide de sens entrouvert par la rupture métaphorique, par des métaphores qui trouvent leur articulation d'un procès de subjectivation.

Le propos d'Aristote cité en exergue est exemplaire à cet égard : nous avons un goût prononcé pour les images, tout particulièrement lorsqu'elles représentent des corps que la vie a quittés ou dont les particularités anatomiques sont choquantes. La représentation aristotélicienne détermine ce qui est propre à ces corps, c'est-à-dire ceux-ci pour autant qu'ils ne sont pas souillés au point de devenir sans-nom. Lorsque ces corps sont bestiaux et mortifères, la forme propre se dégage plus nettement de la matière : on distingue mieux en quoi le connaître informe le regarder, ou ce que le regarder met à portée du connaître. Les analyses de Pierre Gravel rejoignent cette problématique de la représentation dans le moment où elles s'articulent comme pratique de l'impropre: elles veulent jouer d'un écart dans la représentation, soit donc par l'inadéquation de nos représentations, soulever le doute de l'irreprésentable 2 .

Ce qui - dans un récit - nous rapporte au réel c'est ce qui dans le récit lui-même marque que le récit présent n'est qu'une répétition de lui-même, une répétition généralisée qui persiste dans le langage ${ }^{3}$. Il n'y a pas de localisation (rapport d'un $S$ à un $\mathrm{s}^{4}$ ) avant le jeu des ressemblances $(\mathrm{S} / \mathrm{S})$. Mais comment penser la ressemblance sinon par opposition à la localisation et sans une métaphore première dont le premier terme est l'exigence d'un réel qui ne soit pas par avance une figure? Cette analyse requiert le réel comme terme premier mais tout à la fois elle dissout toute localisation dans un langage qui produit la vérité comme effet de déplacement. C'est toute la préoccupation de cette pratique de l'impropre que de déplacer les définitions ${ }^{5}$, et de réduire les écarts, comme une inquiétude qui subsiste et dont on

2. Dans cette étude critique nous essayons de trouver une continuité dans un texte fait de digressions, esquives... Pourquoi organiser un texte membra disjecta si ce n'est supposer derrière celui-ci une méthode: que nous appelons ici la pratique de l'impropre. Il nous suffit de lui prêter une cohérence en formulant un doute : que c'est à toute cohérence que le texte de Pierre Gravel a voulu échapper.

3. Pierre Gravel supposera l'existence de "formes élémentaires de rationalité " qui se répètent autant dans l'ordre narratif que dans l'ordre événementiel et marquent l'un et l'autre comme procédant d'une même répétition (cf. p. 51). Le rapport au réel institué par le récit est alors repérable dans ses parties: ce qui est donné comme répétition dans la chaîne du discours est reçu comme transport ou déplacement dans l'ordre des figures. Mais il ne reviendra pas sur cette notion.

4. $S=$ signifiant, $s=$ signifié selon la réécriture de lalgorithme saussurien $\mathrm{S} / \mathrm{s}$.

5. $\grave{A}$ une définition "naïve» (littéraire) du récit comme copie (qui sert à épingler Genette, p. 50), Pierre Gravel substitue une définition plus avertie (philosophique) du récit comme répétition. Dans l'écart entre les deux, il discerne un «superbe passage» (p. 51). En effet il est question du récit mais c'est du saut par lequel il reformule la définition du récit et de sa définition qu'il s'agit. 
se libère par de nouvelles inquiétudes. Car le gouffre (l'écart) ce n'est pas le réel sous les mots mais l'écart entre deux formules.

Mais tout à la fois, en insistant sur le déplacement «erratique» de la représentation, il semble aussi que ces analyses cherchent également à trouver le terme premier auquel elle s'est substituée. La pensée occidentale serait à portée de sa frâicheur, de l'appréhension première qui l'a occasionnée, sitôt qu'elle se ressaisit dans le mouvement qui la déplace incessamment. Disons-le tout de suite, nous le développerons assez tôt, il s'agirait alors de réduire toute entreprise théorique à un mouvement de métaphorisation qui se poursuit et reste à jamais inachevé, à des métaphores toujours inscrites dans une théorie générale de l'analogie de l'être.

La philosophie se soutient du statut de représentation, comme autonarration qui ne dépend pas d'un narrateur extérieur, récit dont l'illusion diégétique est complète mais ne se laisse reprendre par un autre récit, soit le réel comme récit dont l'illusion est si complète qu'il se méconnâ̂t lui-même comme récit. Le champ est libre pour qui veut faire du théorique une fiction (ou encore subvertir le théorique en tous points par la fiction) puisqu'il ne s'agit que d'œuvrer toujours à cette reconnaissance d'une liberté absolue de la pensée ${ }^{6}$.

En fait c'est une liberté que l'on prend mais qui n'est pas accordée: ne pense pas qui veut et sans contraintes. Il y a une détermination de la pensée sans laquelle elle n'est que simulacres. Le comble c'est de présupposer que cette détermination c'est la philosophie elle-même: Pierre Gravel avait commencé par faire remarquer qu'il y a des séquences de faits parce qu'il y a déjà des séquences de mots (il y aurait déjà du récit). Il tient ensuite les événements comme récit sous le récit, récit subreptice que l'on fait passer pour des choses parce qu'on prétend à la vérité. Mais un renversement est possible: c'est le discours philosophique lui-même qui est déposé dans l'événementiel, il y a un théâtre platonicien qui opère dans les réalités comme dans nos représentations.

\section{VOIR PAR D'AUTRES YEUX}

Dans les métaphores, non seulement on voit par les yeux des autres mais on se donne la possibilité de faire l'expérience originelle qui fonde la pensée. Remarque intéressante: faire une métaphore c'est (comme) voir par ses propres yeux, les métaphores s'usent ou s'enfouissent dans la langue comme reprise de vue. Il fallait qu'en tout premier quelqu'un voie et nomme tout à la fois, pour qu'après on puisse nommer sans voir. Ces métaphores attestent d'une métaphysique de l'image (depuis Aristote) qui a fait du visuel le sens privilégié de tout rapport au réel: le langage trouve sa pleine charge expressive lorsqu'il est un «donner à voir» (p. 101). La métaphore

6. «Si par la métaphore, je vais de lieux en lieux, de cette page, par exemple, à ce qui est suggéré en elle, associer est d'emblée illimité.» $4^{\mathrm{e}}$ de couverture. 
tout à la fois fait surgir l'impropre et conduit à un rapport immédiat à soi-même et à ce qui nous entoure: mais peut-être devrons-nous tenir compte d'un détournement de la métaphore, de l'apparition d'une nouvelle structure spéculaire où ce rapport immédiat se trouve obvié.

Il n'importe donc pas de vérifier l'exactitude des références de Pierre Gravel au monde grec: son intérêt porte d'abord sur la métaphore comme irruption de l'impropre qui génère un sens. Cependant on ne peut considérer la métaphore comme effet de rupture sans tenir compte de l'effet de translation (construction de la référence sur le schéma d'une métaphore première) qui institue le propre à partir de l'impropre et de l'horreur du sans-nom, et tout à la fois retient le propre comme terme premier, comme le terme à partir duquel la philosophie n'a eu de cesse de parler.

Le déplacement nécessite l'usage d'un impropre que l'on met en place d'un propre: jusqu'à ce que l'impropre ne nous apparaisse plus tel, quand les métaphores sont «passées» dans la langue. D'une certaine façon, on conçoit alors que ne nous apparaisse comme significatif que ce qui est inusité, alors que - tout à la fois - ce qui se tient garant de la tradition de la pensée échappe à l'usage. Les cultures se transforment, les formes de conscience se déplacent dans un espace ouvert par la pratique de l'impropre.

D'où la position ambiguë des analyses de Gravel : le langage se prévaut de la puissance de l'image et pourtant, là où le mot semble désigner le plus immédiatement la chose, il n'y a que des effets de déplacement : c'est le port (phora) du mot, le mouvement qui le traverse, qui lui donne sa richesse d'expression comme effet d'un décalage, faisant de celui-ci un «autre-quiconserve-son-étrangeté» (allotriou, cf. p. 101). C'est là tout le mouvement de la signification comme déplacements et écarts dans le signifiant alors que « le sens ne se joue que de tels transports» (p. 7) ${ }^{7}$. La lecture des textes doit ressaisir ce mouvement, sans quoi le texte reste immobile, comme ces textes auxquels rien ne renvoie et dont personne n'emprunte les renvois vers d'autres textes, dans lesquels chacun croit pourtant trouver l'assise de son savoir ${ }^{8}$.

7. Cf. aussi p. 108 « le sens peut se transporter et se manifester comme effet de sens». Ici le texte est tronqué, il faut le compléter en consultant sa première version, parue dans Protée, 1984, p. 44.

8. Avant d'aller plus loin il faut signaler quelques problèmes liés à l'usage de la notion de métaphore. On aura remarqué que cette notion joue sur plusieurs niveaux : I comme mouvement dans l'histoire (p. 106-108), II - comme représentation (le récit comme organisation des faits est lui-même métaphore), III - au niveau de l'expression (sur l'identification de ce niveau avec le précédent, cf. ARISTOTE, op. cit., p. 367), IV - et au niveau de la signification. Si on considère seulement ce dernier niveau, celui de la substitution de mots : déjà le schéma n'est pas clair. La métaphore est d'abord présentée comme un nom qui n'est pas employé en propre, dans son emploi courant, dans son contexte normal, que l'on a trans-porté dans un autre contexte. Le porter-sur du nom (S2) métaphorique se fait sur un autre nom (S1), non pas sur le signifié (s) de cet autre nom (voir schéma I). Un signifiant tombe en position de signifié et se trouve élidé: 
Nous sommes confrontés à cette immobilité des textes: depuis longtemps figés dans l'interprétation et il est difficile de trouver ce qu'ils peuvent «encore donner à penser» (p. 101) et il faut se contenter des miettes laissées par les critiques et commentaires précédents. La question qui se pose c'est tantôt de penser le texte d'une façon qui appartienne encore au texte ou de penser ce qui ne cesse de l'alimenter d'un ailleurs et permet donc le sans-fin de l'interprétation : qui permet en tout premier lieu que je commence la mienne. La métaphorisation comme pratique de l'impropre révèle en quoi le texte tient encore sa signification d'un ailleurs.

\section{Les bandelettes}

Nous étions à la minute de l'ultime distinction. Il fallut rapatrier le couteau. Et l'incarnat analogique.

\section{René Char, Aromates chasseurs?}

\section{LA RÉFLEXION PHILOSOPHIQUE ET LE TRAVAIL DE LA CHAIR}

Certes la métaphore introduit le discontinu, est l'effet d'une rupture: doit-on cependant voir dans tout ce qui coupe, perce, déchire, ... et inversement dans tout ce qui recouvre, panse, embaume et emmaillote les contenus privilégiés de la métaphore ? Ce serait donc à une métaphorologie que nous devons la production de l'horreur. La notion de "plasticité signifiante» cautionne 1) une lecture erratique du texte d'Aristote (mais aussi de tous les textes) d'autant qu'Aristote lui-même aurait conçu le langage comme plasticité (p. 104) et comme une signifiance que produit un jeu de l'altérité et du propre ; 2 ) un usage délibéré de la confusion quant à la forme des métaphores: on suppose que leur contenu révèle le sens de l'activité métaphorique ${ }^{10}$.

$\begin{array}{ccccc}\mathrm{S} 2 & S 1 & \mathrm{~S} 2 & \mathrm{~S} 1 & \mathrm{~S} 2 \\ -\mathrm{S1} & \mathrm{s} & \mathrm{s} & \mathrm{s} 1 & \mathrm{~s} 2\end{array}$

Pourtant Pierre Gravel semble revenir sur cette définition, quand la métaphore « fait porter sur un mot ce que porte un autre » (p. 103), c'est-à-dire qu'elle prête à un mot (S1) la signification (s2) d'un autre (S2) (voir schéma II). Une signification s'impose et supplante une autre, les déplacements dans le signifiant n'étant que les effets de ces nouvelles assignations de sens imposées d'en haut. La métaphore devient un modèle de la domination intellectuelle : l'impropre propose un savoir impossible qui affirme néanmoins se constituer comme savoir en occupant une position maîtresse dans le discours, en proposant un parcours que nul autre ne peut emprunter. C'est un savoir qui s'annonce avec l'impossibilité de tout savoir, avec le soupçon de l'irreprésentable.

9. CEuvres, Gallimard, coll. Pléiade 1983, p. 520.

10. On peut envisager au contraire que c'est le schéma qui est construit à partir des contenus privilégiés. Somme toute, la définition de la métaphore utilise une métaphore spatiale. 
Dans la Poétique d'Aristote les images des métaphores qui servent d'exemples nous renseignent sur le travail métaphorique lui-même - «il est si souvent question d'enlever, de couper, de retrancher (la vie, l'œil, etc.) ${ }^{11}$. Métaphoriser c'est donc aussi couper, retrancher et déplacer dans le texte. Le texte d'Aristote, fortement travaillé par les métaphores, rejoint à la fois la déclamation tragique et l'art du chirurgien et de l'embaumeur.

Cette leçon est reportée sur les textes de Platon qui seraient également travaillés par les «métaphores sophocléennes» (p. 68, 50) ${ }^{12}$ quand il est également question de découper, brûler... : les métaphores révèlent un texte qui ne cesse de se faire violence pour contrevenir à l'afflux du sensible. Pierre Gravel énumère des navettes, bandelettes et sparadraps, tarières et percettes, couteaux, fers à cautériser... comme «instruments de la réflexion platonicienne» (p. 64). On trouve en effet la plupart de ceux-ci au début du Cratyle (387b-389e) où Socrate établit que les noms dérivent de la nature des choses en comparant l'action de nommer à celles de brûler, couper et principalement tisser et percer. Aux développements d'un exemple doivent correspondre des développements équivalents dans les autres exemples. Le tisserand tisse en démêlant les fils avec la navette que le menuisier a fabriquée à partir de l'idée de navette et en tenant compte du genre de tissage. Ainsi l'instructeur nomme en distinguant la réalité avec des noms que le législateur a faits à partir du nom en soi et de façon appropriée à chaque chose. On retrouve donc la plupart de ces instruments sauf les bandelettes et les sparadraps qui servent «à ouvrir la question de l'origine du langage» (p. 64). De toute évidence ils résultent d'une condensation, par Pierre Gravel, entre brûler et couper d'une part et tisser d'autre part : à la confusion des niveaux de la métaphore s'ajoute la confusion entre les métaphores du langage ${ }^{13}$.

11. Jacques DerRIDA, Marges de la philosophie, Minuit, 1972, p. 289, cité p. 69. La phrase se termine comme suit : " tous les termes sont néanmoins présents ou présentables.»

12. Il y a un effet purgateur de l'élision métaphorique. Au corps plein de l'État, ravagé (vidé par en dedans) par la peste ou la guerre civile, se substitue la surface trouée de l'individu (cf. 69-76), la cavité orbitale d'CEdipe, le tombeau béant d'Antigone. L'État platonicien comme corps idéalement plein face aux individus et à leur infinité de manques et défauts constitutifs, devient momie, cocon, etc., vide qui est devenu lieu de prolifération et de dégoût.

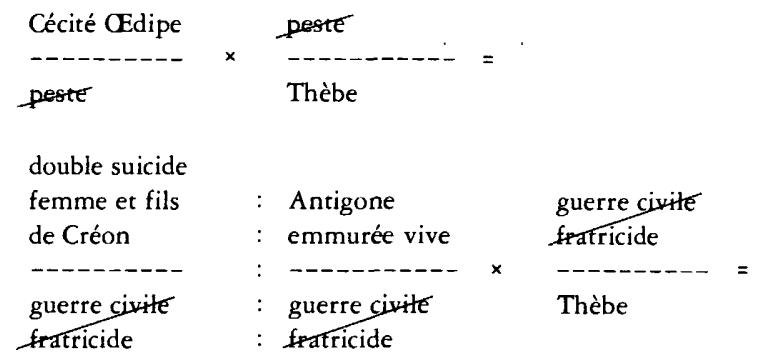

13. Plutôt que d'une condensation, il s'agit d'une ambivalence, ouvrir la plaie et la recouvrir d'un sparadrap relèvent d'une même attitude. Ainsi la pratique de 
Le cratylisme de Pierre Gravel ne serait qu'une naïveté si on ne prêtait attention à la thèse principale de l'origine du langage : elle est suspendue à l'autorité d'un législateur faiseur de noms (qui sait mieux que quiconque «la forme de nom propre à chaque chose» 390a) et d'un dialecticien qui en connaît l'usage convenable. L'insistance du rapport entre faire des noms et faire des instruments à percer, à couper, panser, etc. caractérise ce pouvoir du législateur-dialecticien qui sait comme nul autre (il est «le plus rare parmi les hommes» 389a) ce qui est le propre (il produit un découpage de la réalité) et tout à la fois se permet tous les déplacements, renouvelle le sens dans une pratique délibérée de l'impropre (il sait aussi confondre les choses pour les rendre présentables).

$\mathrm{Au}$ thème de l'interdit platonicien comme expression d'une prise de pouvoir des philosophes sur les affaires de l'État, s'ajoute celui du pouvoir des dialecticiens sur le langage : dès lors qu'il n'y a qu'un seul discours, on ne peut voir autrement, il n'y a pas d'autre voie dans la conduite de la société politique ${ }^{14}$. Les représentations reçues en société, les effets de présence à partir desquels nous constituons un monde, ne sont toujours que ce qui a été rendu approprié, qui se donne sous le couvert du présentable.

\section{PANSER, EMMAILLOTER, MOMIFIER, BANDER}

La reprise d'une philosophie tragique amorcée chez Pierre Gravel présente cette ambiguïté : à la fois panser, répandre les aromates mais aussi laisser libre cours au déplacement, ménager l'impropre en découpant, tranchant, perçant, etc. : à la fois remède et poison. Le spéculatif est toujours boursouflé par le métaphorique ${ }^{15}$, le philosophique est débordé par le littéraire dans le projet de l'homme moderne d'articuler une conception de soi: le travail métaphorologique de l'incision et de l'emmaillotement s'exerce d'abord sur soi, «si on me cautérise ou incise, je veux sentir» disait Montaigne ${ }^{16}$. L'infection se propage d'autant que l'on tâche de l'arrêter : le sparadrap ${ }^{17}$ en tant que remède est aussi un poison (la démonstration du

l'impropre joue de l'écart comme une coupure, et tout à la fois use des bandelettes pour cacher l'écart entre les définitions (p. 52). Noter que les bandelettes qui cachent l'écart entre les définitions (cf. p. 52) sont aussi les bandelettes qui couronnent les poètes (cf. p. 53) : ils font leur gloire à confondre les définitions?

14. C'est sans doute cette perspective qui a occasionné cette synonymie sur « voie»: Paul Mazon (cité dans la bibliographie de Pierre Gravel) donne cette traduction d'un passage de Sophocle dans Antigone (v. 683) «Il se peut cependant aussi qu'un autre voie juste parfois.» (Les Belles Lettres, 1972, p. 117). Pierre Gravel cite ce passage «il se peut cependant qu'une autre voie soit juste parfois» (p. 64).

15. «La pensée spéculative use des ressources métaphoriques du langage» Paul Ricceur, La métaphore vive, Seuil, 1975, p. 395.

16. Essais, Livre II, chap. 12, Gallimard, coll. Folio, 1973, p. 209.

17. Le sparadrap en tant que porter-sur (epiphora) la plaie est le modèle de la métaphore. «Sparadrap» ne figure pas dans les textes grecs (ce terme appartient au vocabulaire médical du latin médiéval) et il y aurait peut-être lieu de parler de l'épithème : «ce qui se place sur», nom donné aux onguents. 
pharmakon chez Derrida), car ce qui couvre, ce qui cache et favorise les proliférations : parler «sur» les choses et surtout parler «sur» les textes anciens c'est aussi se proposer d'écrire ces textes comme pour la première fois, ou du moins de les réécrire.

Aussi, dans son expérience de soi, le moderne spécularise tout, soumet tout le langage à la métaphoricité ${ }^{18}$ pour en attaquer le projet de vérité qui veut que le nom soit propre à la nature de la chose et que le réel soit absorbé dans le langage. En effet l'image en miroir est fidèle mais volatile, fait surgir une étrangeté, une impropriété et «rend impropre le propre» ${ }^{19}$. Avec la métaphore c'est un non-langage qui fait irruption, c'est un extra-langage qu'est le langage du sexe métaphorisé par le signe. Le discours ne réalise pas son identité à soi, il est toujours ouvert par des transports, des déplacements, des transferts: la réponse à l'angoisse s'opère par la sexualisation comme dérivation des corps, la «carnation» comme déplacement des mots.

Le désir de littérature se morfond dans le prétexte philosophique, les désirs inassouvis engendrent la pestilence (William Blake) : ce qui fermente et infecte, c'est le littéraire non par sa nature mais de ce que le philosophique ne saurait lui laisser libre cours. $D^{\prime}$ un miroir... serait un texte philosophique dans lequel s'arquent et tendent, cherchent à s'écrire en même temps plusieurs textes littéraires ${ }^{20}$, et en premier lieu un «texte érotique». On voit en effet Pierre Gravel élaborer le schéma de la métaphore pour produire une mise en scène du corps féminin : telle la surface virginale du corps d'Iphigénie en Aulide (à laquelle Aristote reprochait d'être trop pure pour être vraiment tragique) qu'il suffit de pointer du doigt pour défleurer et tout à la fois marquer d'une plaie. Seul le sparadrap saura restituer à ce corps tout son charme, quand - de bander la plaie sexuelle - il la désigne alors même qu'il la cache. Reste une fascination pour le moribond, la dégradation du corps qui est à notre insu et aussi le maintien de cette fascination dans notre manière d'habiller la mort ${ }^{21}$ et de penser le vivant : c'est la philosophie tout entière qui est devenue un fétiche que l'on fait courir sur le corps perdu, comme tentative tragique de sortir de l'impasse sexuelle.

Les sparadraps et bandelettes doivent être rangés, par leur emploi, dans la catégorie des vêtements de corps affriolants, comme fétiches d'un

18. «La métaphoricité joue avec la fraction du miroir, mais aussi avec le rapport de ces fractions à l'unité du miroir». Luce IRIGARAY, Parler n'est jamais neutre, Minuit, 1985, p. 184-185.

19. Ibid., p. 153.

20. Pourtant la présentation philosophique est maintenue : ce langage du transport, cette parole qui trouve son sens de faire jouer l'autre, est d'abord reconnu par Pierre Gravel comme étant «le lieu de travail de ce que l'on appelle littérature.», Protée, 1984, p. 44, formule importante qui manque dans $D^{\prime}$ un miroir..., p. 108.

21. Un héraut thébain exhorte Thésée : "Romps le charme des saintes bandelettes.» Les suppliantes, $449 \mathrm{et} \mathrm{sv}$. Cf. Thomas STERN, Thésée ou la puissance du spectre, Seghers, 1981, p. 249, n. 1. 
désir insupportable qui viennent nier la coupure, la séparation qui nous met à distance de la nature : à ce chapitre Pierre Gravel nous invite à la prudence dans la garde-robe, « une robe funéraire n'est pas un sparadrap, une robe de mariage pas un vêtement de corps» (p. 65). Le vêtement peut à la fois cacher la plaie (le putride et le bestial : la « réalité » du corps) et m'assurer de ce qui m'est propre (mon sexe signifié) quand il aura su lui donner une forme (qui le dénature). Le fétiche maintient l'identification au monde: les mots sont des choses, du moins s'y substituent complètement.

Ce ne sont pas seulement la République de Platon (p. 69) ou l'État (p. 76) mais aussi tout le discours philosophique qui font office de fétiches, qui se sont substitués au monde pour l'avoir «couvert» exhaustivement. $C e$ ne sont que des «momies», lorsqu'il aura été du destin de la pensée de dépasser l'appréhension première (la saisie mais aussi l'horreur qu'il a fallu d'abord surmonter) pour déployer un logos dont la cohérence nous fait reconnaître en celle-ci l'exercice même de la pulsion de mort. Penser c'est panser (cf. p. 65) le vif et c'est aussi apprêter (emmailloter) le mort. Reconnaître ce qui est le propre de chaque chose, faire ce qui est approprié : voilà qui nous installe d'emblée dans la mort. La pensée n'est toujours qu'une nature que nous avons su capter mais que nous avons aussitôt dénaturée, c'est une mort que l'on sait entourer d'aromates.

Ainsi le soupçon de l'irreprésentable s'est-il maintenu sous la forme d'une pratique de l'impropre. La continuité d'une telle pratique se donne à prendre le relai des métaphores : penser c'est filer la métaphore, c'est tisser les représentations : la métaphore du langage comme tissage est première. Cependant Pierre Gravel revient toujours sur ses préoccupations en affirmant que «tout se passe comme s'il pouvait s'agir de panser une plaie - qui ne sera jamais pourtant nommément désignée - ou de momifier quelques cadavres absents.» (p. 65). Le cadavre est absent comme le sans-nom fait défaut sitôt qu'il y a nomination.

Pourtant l'horreur soutient la réflexion comme chirurgie et embaumement, car il y a une cruauté, " une blessure qui est infligée et qui dans le texte dit "métaphysique", n’est jamais nommément désignée» (p. 70) mais qui représente (répète) ce qu'il en coûte d'instaurer le métaphysique. Il y a une genèse de la pensée (son pouvoir imageant et ses images imprésentables) qui persiste dans son expression dernière (sa formulation abstraite), il y a un impensable qui demeure dans la pensée même et dont celle-ci ne cesse de s'exciser. La pensée n'a pas rendez-vous avec le monde, elle n'a de cesse de résoudre sa béance.

\section{Les miroirs}

En ce temps-là, le monde des miroirs et le monde des hommes n'étaient pas comme maintenant, isolés l'un de l'autre. Ils étaient, en outre, très différents; ni les êtres ni les couleurs ni les formes ne coïncidaient.

$$
\text { Jorge Luis Borges \& Margarita Guerrero }{ }^{22}
$$

22. Manuel de zoologie fantastique, trad. G. Estrada \& Y. Péneau, UGE, coll. 10/18, 1970, p. 15. 


\section{L'IMPORTATION DES MIROIRS}

Le sens résulte d'un jeu de l'altérité dans le langage et aussi dans la société. La philosophie elle-même serait vouée à toujours parler à partir d'un ailleurs. Il apparaît alors significatif qu'Aristote soit un étranger dans la Cité (p. 105). La métaphore constitue le modèle de cette advenue d'autrui, elle est importation, im-migration du sens qui suppose un sens autochtone.

Pierre Gravel nous rapporte un scénario exemplaire de cette importation de sens: d'une culture, d'une domination. On y voit le miroir jouer comme métaphore d'une société, comme point d'appui de la métaphorisation d'une société par une autre:

« les premiers blancs qui sont arrivés en ces terres d'Amérique ne s'y sont pas trompés en distribuant massivement de tels instruments de réflexion qui ont littéralement séduits des populations entières. Avant le miroir, aucun Indien ne s'était vu» (p. 158)

Pour Pierre Gravel, se civiliser c'est importer des miroirs et surtout ce miroir massif qu'est le langage : on voit qu'il ne s'agit pas d'alphabétisation ou de séduction par le littéral mais d'un mouvement interne au langage, d'un pouvoir de translation qui en fait une force civilisatrice. La métaphore est en ce sens déplacement et domination. La métaphorisation (et aussi la spécularisation) est le processus de la civilisation. Le mouvement de l'occidentalisation (p. 66) commence avec le développement de l'expérience réflexive dans la philosophie occidentale à partir du «je pense» de Descartes qui, il faut le croire, ne méditait pas dans son poële mais devant un miroir car, selon Pierre Gravel, il n'y a pas de réflexivité sans miroir (cf. p. 158).

Occidentaliser, c'est faire entrer dans la modernité. Pour cela il ne suffit pas de distribuer des miroirs, encore faut-il disposer d'une structure spéculaire qui permette une certaine reconnaissance de l'image en miroir, ou du moins de la recevoir comme telle, sans néanmoins réintroduire la figure de l'Autre (Dieu, la communauté). Dans l'articulation auto-référentielle que permet le miroir persiste une recherche de l'Autre et le refus de la reconnaissance par l'autre. C'est toute l'angoisse de la Modernité que de vouloir se donner une expérience de son être propre sans autre référence que sa propre image et sans parvenir à ce face-à-face alors qu'elle ne parvient pas à s'échapper de la structure spéculaire classique.

\section{MÉTAPHORE ET MIROIR : LA STRUCTURE SPÉCULAIRE CLASSIQUE}

La métaphore, comme perception des ressemblances ( to bomeion theôrein», Poétique 59a8) est aussi le miroir: ce rapprochement éclaire considérablement la lecture de D'un miroir... où la perception dans le miroir produit un effet de sens. Le premier terme de la ressemblance n'existe que parce qu'il y a perception de la ressemblance, que son image ne s'y constitue 
pas à partir d'une identité propre mais à partir d'un jeu relationnel où elle rassemble ses mille éclats.

Le rapport spéculaire redouble le signifiant d'un signifié (deux $\mathrm{S}$ pour un s). L'image dans le miroir est la métaphore d'une identité instable. Redoublé par un S spéculaire le sujet se découvre par avance comme pur $\mathrm{S}$ (ou reflet): se perd comme identité (le fait même qu'il existe voudrait dire quelque chose). C'est dans cette expérience de déprivation, où l'identité reste suspendue comme un simulacre, que les $\mathrm{S}$ renvoient les uns aux autres et ne se juxtaposent pas tout simplement.

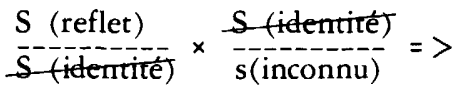

Le miroir est cette arète vive où se constitue le sujet pour autant qu'il découvre qu'il est aussi un autre pour les autres ${ }^{23}$. L'identité ne se constitue que dans l'ouverture d'une altérité. La métaphore dans le langage marque l'écart du sujet, son altérité et son impuissance à rejoind re la position stable du «Je» de l'énoncé. Le sens est posé et dérobé dans le texte comme fragmentation du sujet, comme son miroir en éclats.

Le miroir nous révèle que nous sommes pur reflet: le leurre de s'appartenir vole en éclats. Pourtant le sujet n'est pas volatilisé, il n'est que démultiplié en reflets périlleux ${ }^{24}$. On est encore pris avec soi-même, on se découvre nanti d'une existence que par et avec un corps. Épreuve tragique, ce n'est que par l'unité du corps que nous rassemblons l'expérience morcelée de notre existence. Or c'est bientôt le corps lui-même qui vacille et devient le lieu d'une hétérogénéité radicale.

\section{LA SUBJECTIVITÉ TRAGIQUE}

Alors qu'est-ce que le tragique si ce n'est le devenir autre dans son $\operatorname{corps}^{25}$, la dispersion et l'aliénation du sujet? Échappée de soi d'autant plus subversive que l'ordre social de la République serait fondé sur l'assignation ponctuelle de chacun à une identité. Je deviens autre parce que je me découvre reflet, et fais vaciller l'identité de mes concitoyens. La pratique de

23. "Le miroir est une machine en, par et à travers l'opacité de laquelle "je" puis "me" structurer comme un autre pour tous les autres : ce en quoi consiste le travail du maquillage.» p. 158.

24. Il serait utile de tenter d'inscrire cette problématique du reflet en rapport à l'histoire de la philosophie : la référence à Leibniz s'impose ici, qui nous découvre la monade comme représentation («miroir vivant» disait Leibniz) dans laquelle se reflète tout ce qui des autres monades est également reflet. Le monde dès lors n'est fait que d'éclats. Cf. Catherine Clement, Miroirs du sujet, coll. 10/18, 1975, p. 24-25, 202.

25. « Devant un miroir (...) je vois (...) ce visage aux traits ravagés par le travail du temps ou la fatigue intemporelle des nuits sans sommeil.» p. 158. 
l'impropre chez Pierre Gravel devient ici recherche et aussi contestation de l'identité : elle s'apparente au jeu de l'acteur (l'bypocrytès) qui se grime et se maquille (cf. 76, 158). Pierre Gravel veut pousser plus loin cette recherche: «Je dois être une autre» (p. 75). Voilà le sujet non seulement maquillé mais travesti ${ }^{26}$. À un certain lien social correspond un certain refoulement sexuel. Il faut contester ce lien jusqu'à l'hétérogénéité radicale qui se découvre en nous d'abord comme plaie sexuelle inguérissable, comme mode de subjectivation irréductible.

Sujets en sursis, nous devenons acteurs sur un théâtre dont Artaud disait qu'il «doit s'égaler (...) à une sorte de vie libérée, qui balaye l'individualité humaine et où l'homme n'est plus qu'un reflet ${ }^{27}$. " Le tragique, est-ce le théâtre pauvre de l'existence quotidienne où l'homme n'est même pas un reflet mais seulement un fragment ${ }^{28}$. Ou bien encore le tragique est-il ce moment où l'homme se dépasse en niant son nom et son sexe? Voilà que pour cet athlète affectif, les accessoires de théâtre et les articles de la toilette féminine (cf. p. 75) se confondent pour faire de lui un de ces «hétéro-somato-phores» (p. 159) qu'il nous faut devenir : ceux qui portent dans l'autre le somatique ${ }^{29}$.

\section{L'ÉPREUVE DE LA SPECCULARISATION}

L'altérité, la non-identité à soi est un passage nécessaire pour s'approprier l'image en miroir. Pour accéder à l'identification il faut reconnaître l'image en miroir comme autre pour qu'elle soit aussi la même : l'exigence spéculaire moderne demande qu'elle ne soit autre pour un autre mais seulement dans l'écart ouvert par le fonctionnement inapproprié du moi. On peut identifier cette difficulté de maintenir une cohésion du moi à la figure troublée de l'hystérique telle que révélée par la clinique ${ }^{30}$. L'épreuve de la spécularisation sera toujours refusée chez l'hystérique: celui-ci, toujours voué à une identité à soi précaire, dérisoire, n'accepte une image «qu'en tant que fragment, facette d'une unité toujours à venir » ${ }^{31}$. D'une

26. Il aurait été intéressant dans ce contexte de se situer par rapport aux travaux de Marie Delcourt sur l'hermaphrodisme. Pierre Gravel mentionne son nom (p. 63) et non ses ouvrages: il s'agit d'CEdipe ou la légende du conquérant, Belles Lettres, 1981, et surtout de son Hermaphrodite: mythe et rites de la bisexualité dans l'antiquité classique, PUF, 1958.

27. Cuvres complètes, Gallimard, 1978, IV p. 112.

28. "Chelovek (human being) must know where he stands and let others know, otherwise he is not even a klok (piece) of a chelovek, neither a he, nor she». Vladimir NaboKov, Ada or Ardor: A Family Cbronicle, Fawcet Crest, 1969, p. 33.

29. Phorein : c'est aussi porter l'enfant, être en puissance d'enfanter. Genos : la filiation, la souche d'une naissance, d'une origine, d'une famille (cf. Guy BouCHARD, Le procès de la métaphore, Hurtubise $\mathrm{HMH}, 1984$, p. 19). La métaphore du genre à l'espèce ou de l'espèce au genre, c'est la reproduction dans laquelle nous sommes des acteurs indifférenciés dont le sexe change et se déplace (allotropique).

30. Masud Khan, Passion, solitude et folie, Gallimard, 1985, p. 54.

31. IRIGARAY, op. cit., p. 29. 
certaine façon, son miroir est toujours brisé, il se sent incapable de comparer son image au père législateur, au père porteur de la Loi et fondateur des significations, en regard duquel il s'éprouve insignifiant. Alors il se peut qu'éprouvant que son image est trop pauvre (elle est moins que reflet) il brise lui-même le miroir pour ne pas l'y laisser. Cette image, il n'aura de cesse de la contester, la récuser et redoute d'être arrêté dans une identité (dans un éclat) parce qu'il vise un objet idéal (le miroir) : son drame tient à la confrontation d'un miroir et de quelques éclats. Car il ne peut attester son image par son seul regard et ne se convainc pas d'exister dans le regard de l'autre.

Insaissisable pour lui-même, il rencontre partout son image spéculaire, soutenue par autrui pour qu'il s'y confronte. Bien qu'il soit lui-même le sujet de cette représentation, celle-ci lui paraît n'être avant tout qu'un objet pour l'autre et il ne la reprend pas à son compte. L'hystérique en quelque sorte dit : - Je ne fais que passer, ce que vous dites de moi (ce que vous entendez de ce que je dis de moi) n'est qu'une projection de votre part. Cette image, je n'en ai pas la responsabilité. De mes écrits, vous pouvez dire ce que vous voulez ${ }^{32}$.

Le style philosophique de Pierre Gravel apparaît comme un symptôme de ce sujet moderne qui ne peut dès lors que se maintenir dans le morcellement du miroir, les identifications hétérogènes, les spécularisations incomplètes, et se re-spéculariser sans cesse. Il est toujours en train de naître, il dit tout à la fois qu'il n'existe pas comme sujet ${ }^{33}$, ou du moins qu'il n'est pas intéressant comme sujet, quand le monde reste à découvrir et tout reste à dire. Car les significations qu'il pourra donner ne seront jamais à la hauteur de celles que donnerait en la même occasion un législateurdialecticien, maître du langage, trésorier du signifiant. Il pourra tout métaphoriser, traverser, déplacer, ... il reproduit en tous lieux une même structure spéculaire.

Car, au gré du travail des métaphores, c'est l'image en miroir qui acquiert le statut de transcendance. Si le moderne spécularise tout c'est qu'il est en quête d'un discours total. Gravel perfectionne un style philosophique

32. «L'hystérique ne tarit pas de paroles. Parole pleine, oui, mais qu'il conteste aussitôt. Là n'est pas vraiment ce qu'il voulait dire. Qu'on n'aille surtout pas le prendre au mot! C'est sa hantise. Il reprend le défilé des signifiants, en retenant un pour le rejeter aussitôt et en élire un autre, qu'il renie sitôt émis. C'est sans fin. Car, pas plus que son image ne lui est tolérable, il n'accepte de se déterminer en son discours. Le défilé des signifiants comme celui des images, son discours en zigzag, les masques dont il change à tout propos, disent assez son désir d'un discours total qui engloberait tous les signifiants comme son image serait à facettes innombrables, contradictoires. Et après? Il s'égare dans ses énoncés, ne se reconnaît plus en ses masques, s'angoisse.» Cf. IrIGARAY, op. cit., p. 33.

33. Le tragique ce n'est pas tant l'absence de sujet que l'impossibilité d'échapper au mirage du sujet: «Le moi ne peut s'échapper à lui-même.»Cf. Sigmund Freud, Métapsychologie, coll. Idées, p. 45. 
qui cherche à réglementer la scénographie du sexe ${ }^{34}$ mais qui tout à la fois la déploie de tous côtés. Il y a cette oscillation dans son texte entre le présenter (rendre présentable) et le donner à voir (exhibition de l'horreur). Celui-ci joue de la métaphore mais ne dépasse pas la structure du miroir qu'elle sous-tend : il joue du pouvoir d'abstraction et d'idéalisation de la métaphore lorsqu'elle est commandée par la structure spéculaire classique ${ }^{35}$. L'écart à peine institué est aussitôt repris, sans apporter de modifications dans la structure spéculaire du phallocentrisme ${ }^{36}$, sans produire le déplacement qui permettrait un autre discours sur la Loi, celui que cherchait Antigone.

Lauris-sur-Durance

Département de philosophie

Université du Québec à Montréal

34. Cf. Irigaray, op. cit., p. 176. Il semble que D'un miroir... offre une version moins hérissée des articles colligés : on peut comparer par exemple la version parue dans Brèche, no $4-5$, p. 81 et celle dans D'un miroir... p. 22, où disparaît un «sexe dressant».

35. Cf. Irigaray, op. cit., p. 184, par re-présentation et re-doublement vers le sublime; cf. aussi Derrida p. 269 et 303 de Marges...

36. La structure spéculaire classique est articulée par une métaphore phallique. En effet le nom du père se substitue, dans l'imaginaire du sujet, «à la place premièrement symbolisée par l'opération de l'absence de la mère» (Jacques LACAN, Ecrits, Seuil, 1966, p. 557) et détermine ainsi la signification du phallus :

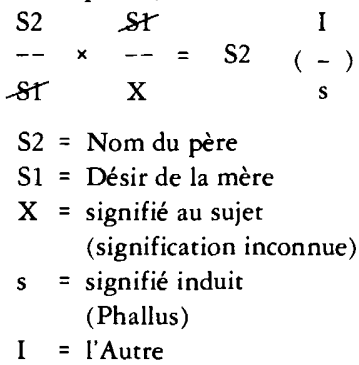

